\title{
NOTE
}

\section{Fractals in ecology: methods and interpretation*}

\author{
R. H. Bradbury ${ }^{1}$, R. E. Reichelt ${ }^{1}$ and D. G. Green ${ }^{2}$ \\ 'Australian Institute of Marine Science, PMB No. 3, Townsville MSO, Australia 4810 \\ ${ }^{2}$ Department of Biogeography and Geomorphology, Australian National University, GPO Box 4, Canberra, Australia 2601
}

\begin{abstract}
We agree that the method of computing fractals described by Mark (1984) is both more appropriate and more informative than the method we used (Bradbury and Reichelt, 1983). In this note, we argue that it is the departure from strict self-similarity in real structures that gives them their ecological interest. We support these arguments with the analysis of new finer-resolution data from the previously studied coral reef which suggests that reefs are extremely smooth structures at the scale of coral colonies, and that this smoothness is reduced at both finer and coarser scales.
\end{abstract}

David Mark (1984) has voiced what we ourselves have also realized. The method of computing fractals that he describes (Mandelbrot, 1977; Goodchild, 1980) is both more appropriate and more informative than the method we used (Bradbury and Reichelt, 1983). However, we feel that there is a risk that Mark's discussion may leave some readers with the impression that lack of self-similarity invalidates the whole fractal approach to ecosystem studies. In this note we wish to emphasize that this is not so; it is precisely the lack of self-similarity that is of greatest ecological relevance and interest.

Problems with variograms. The variogram approach to fractal estimation assumes that one is dealing with a continuous function $W(t)$ of some independent variable $t$. In this context, the only possible interpretation of $t$ is 'straight-line' distance along a transect, while W must represent the corresponding distance measured along the reef surface. Fractal estimates are then based on the variance of increments

$$
\mathrm{V}(\mathrm{T})=\mathrm{E}\left\{|\mathrm{W}(t)-\mathrm{W}(t+\mathrm{T})|^{2}\right\},
$$

where $\mathrm{T}=$ step length used (Berry and Lewis, 1980). The chief practical difficulty is that in the reef transects, our steps with dividers were made along the reef surface, whereas $\mathrm{T}$ in this context represents increments in the straight line distance. A further problem is that $V(T)$ should be computed over all values of $t$ (or at

\footnotetext{
- AIMS Publication No. 211
}

least be estimated from some representative subsample), whereas with our sampling method we effectively used only those values of $t$ that are multiples of $T$, thus introducing a systematic bias into the results.

Ecological interpretation of fractal dimensions. True fractals are abstractions; no real-world phenomenon is self-similar at all scales. Individual biological (and other) processes always operate over a restricted range of scales. However, the geometric properties of the patterns they produce, if extended over all possible scales, would define true fractal curves. Thus estimates of fractal dimension made over the restricted range of scales do reflect the geometric properties of the processes. Peaks and dips in the value D of this 'fractal index' (our term for the curve showing estimates of fractal dimension against scale) indicate shifts in the sources of biological pattern. The fractal index is thus a statistical tool for examining patterns and processes in coral communities.

Estimates of D for real processes (whether obtained by the variogram or ratio method) may stray outside the range 1.0 to 2.0 in certain circumstances. Such transgressions should not be interpreted as evidence that the application of fractal theory is not valid. They might, on the contrary, provide useful information about the phenomenon concerned, for they may indicate limits to the range over which particular processes affect the system (growth of individual corals, for instance).

Reanalysis of our data. We have now reanalysed the data presented in Bradbury and Reichelt (1983), together with a more comprehensive suite from the same site (Fig. 1) which we have since collected. These new data range over 7 scales, with a finer degree of resolution than we were able to report before. Since the method recommended by Mark allows us to make estimates of $D$ at each scale, we are now able to examine the variation of $D$ both within-scale and between-scale as well as with the finer resolution 
offered by the new data. We consider our new data set to be superior not only in terms of resolution but also in terms of accuracy (as will be explained below). Therefore we will focus on these new data in the remainder of this reply.

Within-scale variation of $D$. We have split our samples in the following ways to obtain additional information on the within-scale variability of $\mathrm{D}$ : (1) In order to examine the stationarity (or stability) of $\mathrm{D}$ at each scale, the sequence of observations at each scale was split into 2 subsequences that join end-to-end. (2) In order to examine the local variability of $\mathrm{D}$ at each scale, 2 interlaced subsequences were formed by selecting alternate observations from the original sequence of data.

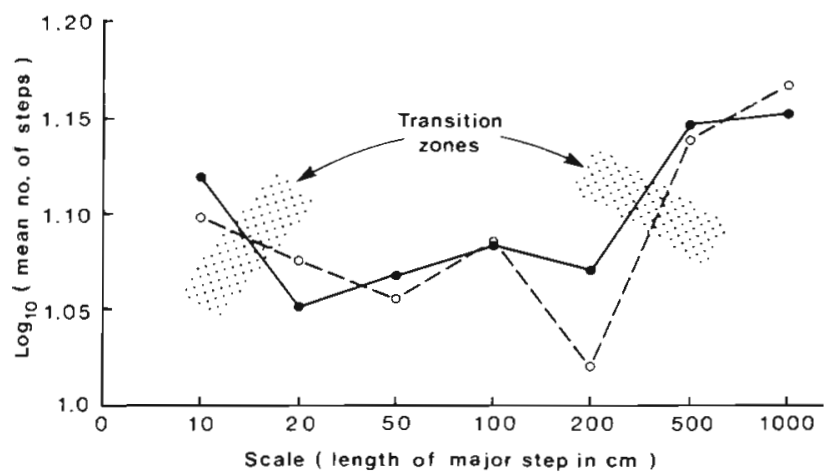

Fig. 1. Fractal index for 2 interlaced sequences measured at each of 7 scales on Myrmidon reef crest

Fig. I shows the interlaced results, which imply that the local variation in $\mathrm{D}$ is small. The stationarity results (not shown) were similar, implying that the process measured by D was stationary. These results show that the within-scale variation of the fractal index is smaller than systematic between-scale variations. The between-scale variations therefore indicate real changes in the processes causing pattern in the reef surface. We must stress that data sampling in the reef environment is difficult and time-consuming; the needs of analysis conflict with the demands of field logistics. Thus even our enlarged data set does not enable more rigorous statistical analysis. We are currently examining the problem of statistical testing in this context.

Between-scale variation of $D$. The new results confirm Mark's observation on our earlier data that the realms of $\mathrm{D}$ are low in comparison with other geographical structures such as coastlines (which are typically about 1.2). However, the finer resolution and small within-scale variation implicit in the new data allow us to extend these conclusions significantly. D declines from a value of about 1.1 at the finest scale measured $(10 \mathrm{~cm})$ to a value of about 1.05 at which it remains over next 4 larger scales $(20,50,100$ and 200 $\mathrm{cm}$ ). It then rises steeply to a value of about 1.15 where it remains for the two largest scales (5 and $10 \mathrm{~m})$.

This suggests that the processes generating the reef topography are self-similar within each of two ranges of scales 20 to $200 \mathrm{~cm}$ and 5 to $10 \mathrm{~m}$ and that there is a 'transition zone' (Mandelbrot, 1977) between them. It also suggests that there is a 'transition zone' between the self-similar processes over the 20 to $200 \mathrm{~cm}$ scales and the processes at the finest scale of $10 \mathrm{~cm}$.

These 3 ranges of scale $10 \mathrm{~cm}, 20$ to $200 \mathrm{~cm}$ and 5 to $10 \mathrm{~m}$ ) overlap strongly with the ranges of scales of 3 different reefal structures:

(1) The individual structure of coral colonies (their branches, and convolutions) are in the range of $10 \mathrm{~cm}$; (2) the distribution of sizes of living adult coral colonies are in the range of 20 to $200 \mathrm{~cm}$; and (3) the spurs, grooves, buttresses and other similar geomorphological structures are in the range 5 to $10 \mathrm{~m}$.

The above results lead us to the conclusion that, contrary to Bradbury and Reichelt (1983), coral reefs are living surfaces that are unusually smooth structures in the fractal sense. Moreover, this smoothness is expressed most strangly over a wide range of scales (20 to $200 \mathrm{~cm}$ ) through the coral colonies themselves, the major architects of the reefs. The topography is not so smooth above and below those scales.

Since the major effect of low fractal dimension is to reduce the intimacy of contact of the living surface of the reef with the surrounding water, and since this effect is most strongly expressed at the scale of coral colonies, we now speculate that corals attempt, at the colony level, to reduce the intimacy of their contact with the medium, and that this tendency is countered by processes within colonies and at geomorphological scales that tend to raise the fractal index. We are now investigating these ideas on different reefs and in different reef zones.

\section{LITERATURE CITED}

Berry, M. V., Lewis, Z. V (1980). On the Weierstrass-Mandelbrot fractal function. Proc. R. Soc. Lond. A 370: 459-484

Bradbury, R. H., Reichelt, R. E. (1983). Fractal dimension of a coral reef at ecological scales. Mar Ecol. Prog. Ser 10: 169-171

Goodchild, M. F. (1980). Fractals and the accuracy of geographical measures. Math. Geol. 12: 85-98

Mandelbrot, B. (1977). Fractals: form, chance, and dimension. Freeman, San Francisco

Mark, D. M. (1984). Fractal dimension of a coral reef at ecological scales: discussion. Mar Ecol. Prog. Ser 14 293-294

Accepted for printing on August 11, 1983 